\title{
Enhancement of anti-murine colon cancer immunity by fusion of a SARS fragment to a low-immunogenic carcinoembryonic antigen
}

\author{
Chen-Si Lin', Shih-Han Kao ${ }^{2}$, Yu-Cheng Chen², Chi-Han Li ${ }^{2}$, Yuan-Ting Hsieh², Shang-Chih Yang ${ }^{2}$, Chang-Jer Wu ${ }^{3}$, \\ Ru-Ping Lee ${ }^{4}$ and Kuang-Wen Liao ${ }^{2 *}$
}

\begin{abstract}
Background: It is widely understood that tumor cells express tumor-associated antigens (TAAs), of which many are usually in low immunogenicity; for example, carcinoembryonic antigen (CEA) is specifically expressed on human colon cancer cells and is viewed as a low-immunogenic TAA. How to activate host immunity against specific TAAs and to suppress tumor growth therefore becomes important in cancer therapy development.

Results: To enhance the immune efficiency of CEA in mice that received, we fused a partial CEA gene with exogenous SARS-CoV fragments. Oral vaccination of an attenuated Salmonella typhimurium strain transformed with plasmids encoding CEA-SARS-CoV fusion gene into BALB/C mice elicited significant increases in TNF- $\alpha$ and IL-10 in the serum. In addition, a smaller tumor volume was observed in CT26/CEA-bearing mice who received CEA-SARSCoV gene therapy in comparison with those administered CEA alone.

Conclusion: The administration of fusing CEA-SARS-CoV fragments may provide a promising strategy for strengthening the anti-tumor efficacy against low-immunogenic endogenous tumor antigens.
\end{abstract}

Keywords: immunotherapy, tumor-derived peptide, tumor vaccine, low-immunogenicity

\section{Background}

Tumor-associated antigens (TAAs) are attractive candidates for cancer therapy in view of their specificity to tumor targets and safety profile. Although most known tumor antigens can generate cancer-specific immune responses, they are either poorly immunogenic or functionally non-immunogenic, and may not elicit immune responses sufficient to eradicate tumor cells [1]. Therefore, efforts to enhance TAA-mediated host immunity towards tumors are being made by exploring a variety of adjuvants for immunotherapy, adjuvants for cancer vaccines such as highly-immunogenic vectors, combinations of T-cell co-stimulatory molecules, or immune-stimulatory cytokines usually being employed [2]. Carcinoembryonic antigen (CEA) is a well-known and over-expressed TAA in most human colon carcinomas,

\footnotetext{
* Correspondence: liaonms@pchome.com.tw

2Department of Biological Science and Technology, National Chiao-Tung University, Hsin-Chu, Taiwan

Full list of author information is available at the end of the article
}

and vaccination with CEA has been demonstrated to induce, although with only minor effects, both humoral and $\mathrm{T}$-cell responses [3]. In order to magnify the CEAmediated tumor-specific immune activities, co-administration of CEA with other immune-response enhancers such as granulocyte-macrophage colony-stimulating factor (GM-CSF) [4] or interleukin (IL)-12 [5] has yielded promising results with regards to the activation of cellular and humoral CEA-specific immune responses.

Some antigenic peptides derived from the severe acute respiratory syndrome coronavirus (SARS-CoV) have exhibited strong immunogenicity, and specific immunogenic epitopes (S978 and S1203) of the S protein of SARS-CoV have been demonstrated to exhibit a vigorous affinity towards binding with HLA-A2 and induce high levels of gamma interferon (IFN- $\gamma$ )-secreting $\mathrm{T}$ cells [6]. Therefore, employment of these highly-immunogenic peptides may boost the positive properties of CEA tumor vaccines.

\section{C) Biomed Central}

(C) 2012 Lin et al; licensee BioMed Central Ltd. This is an Open Access article distributed under the terms of the Creative Commons Attribution License (http://creativecommons.org/licenses/by/2.0), which permits unrestricted use, distribution, and reproduction in any medium, provided the original work is properly cited. 
In this study, we constructed fusion genes by fusing a partial sequence of CEA with highly-immunogenic fragments of SARS-CoV, which have been verified consistently to effectively elicit IFN- $\gamma$ secretion in humans [6]. For achieving simple administration of drugs and enhancement of host immunity, an oral DNA vaccine vector, Salmonella typhimurium (SL3261) [7], was used as a carrier to deliver the SARS-CoV-CEA fusion genes. Using these methods, we sought to demonstrate the administration of a customized and efficient cancer therapy that combines any selected TAAs and immune-activating sequences using a simple drug-delivery method. Significant retardation of tumor growth and increased survival were observed in mice with cancers in our study, and the results obtained using this strategy were therefore promising.

\section{Results}

Prediction of H2-Kd binding epitopes from CEA-SRAS-CoV fusion polypeptides

The SYFPEITHI prediction algorithm was used to predict peptide/H2-Kd interaction by affinity calculation. To find a low-immunogenic TAA and prevent the human TAA from activating mouse immunity, a truncated CEA peptide was used as a low-immunogenic tumor antigen in this study, the sequence of which is shown in Figure 1a. The CEA sequence was predicted to be less immunogenic by affinity calculation because its highest epitope score (CEA a.a. No. 5-13 (Figure 1a): epitope score 16) was far lower than the scores of well- known epitopes of Listeria monocytogenes (Listeriolysin O) (LLO91-99: epitope score 24; p60217-225: epitope score 27) (Table 1). A selected SARS fragment was fused with CEA, and this sequence is shown in Figure 1b. As described previously, the epitope within the CEA-SARS-CoV amino acid sequence was also calculated and compared with known epitopes in Listeria monocytogenes (LLO91-99, p60217-225). As a result, the highest score within the CEA-SARS sequence was 21 in the SARS region (Table 2), lower than the scores of known epitopes of L. monocytogenes for peptide/H2-Kd interaction. In addition, fusion of the CEA and SARS sequences did not create a new epitope with a higher score (data not shown).

\section{Effects of tumor vaccines on cytokine profiles}

To examine the cytokine profiles of the different immunized groups quantitatively, sera samples were collected from each group 4 weeks after vaccination (i.e., 2 weeks after tumor inoculation), and cytokines representing Th1 (TNF- $\alpha$,) and Th2 (IL-10) were detected by ELISA. The pCEA-SARS-CoV group revealed significantly higher TNF- $\alpha$ and IL-10 production than the PCEA and control groups $(p<0.05)$ (Figure 2)

Vaccination and therapeutic effects of orally-administered PCEA- and pCEA-SARS-CoV-transformed S. typhimurium

To determine the efficacy of the pCEA-SARS-CoV tumor vaccine, mice were orally immunized with $1 \times 10^{8}$ pCEAand pCEA-SARS-CoV-transformed S. typhimurium 14

a.

$\begin{array}{cccccccccccccccc}\text { ATG } & \text { GAG } & \text { TCT } & \text { CCC } & \text { TCG } & \text { GCC } & \text { CCT } & \text { CCC } & \text { CAC } & \text { AGA } & \text { TGG } & \text { TGC } & \text { ATC } & \text { CCC } & \text { TGG } \\ \text { M } & \text { E } & \text { S } & \text { P } & \text { S } & \text { A } & \text { P } & \text { P } & \text { H } & \text { R } & \text { W } & \text { C } & \text { I } & \text { P } & \text { W } \\ \text { CAG } & \text { AGG } & \text { CTC } & \text { CTG } & \text { CTC } & \text { ACA } & \text { GGT } & \text { GAA } & \text { GGG } & \text { AGG } & \text { ACA } & \text { ACC } & \text { TGG } & \text { GAG } & \text { AGG } \\ \text { Q } & \text { R } & \text { L } & \text { L } & \text { L } & \text { T } & \text { G } & \text { E } & \text { G } & \text { R } & \text { T } & \text { T } & \text { W } & \text { E } & \text { R } \\ \text { GTG } & G G A & G G A & G G G & \text { AGC } & \text { TGG } & \text { GGT } & \text { CTC } & \text { CTG } & \text { GGT } & \text { AGG } & \text { ACA } & \text { GGG } & \text { CTG } & \\ \text { V } & \text { G } & \text { G } & \text { G } & \text { S } & \text { W } & \text { G } & \text { L } & \text { L } & \text { G } & \text { R } & \text { T } & \text { G } & \text { L } & \end{array}$

b.

Figure 1 Flanking nucleotide sequences of the CEA and SARS epitopes. (a) 132 bps (nucleotides 12804 10935) of the CEA sequence (NCBI accession No. Z21818) and (b) 177 bps of the SARS sequence (NCBI accession No. AB263618) were used as the starting materials to generate highly-immunogenic mutations. The grey area indicates the epitope used for affinity prediction. 
Table 1 Comparison of the CEA and CEA-SARS epitopes with other known epitopes that have been proven to elicit immunity in BALB/c mice.

\begin{tabular}{|c|c|c|c|}
\hline Gene & Primer & Sequence $\left(5^{\prime} \rightarrow 3^{\prime}\right)$ & $\operatorname{Tm}\left({ }^{\circ} \mathrm{C}\right)$ \\
\hline & P1 & TACggAATTCATggAgTCTCCCTCggCCCCTCCCCACAgATggTgCATCC & 71.6 \\
\hline & P2 & CCTggCAgAggCTCCTgCTCACAggTgAAgggAggACAAC & 71.5 \\
\hline & P3 & CTgggAgAgggTgggAGggAgggAgCTggggTCTCCTgggT & 75.1 \\
\hline \multirow[t]{7}{*}{ CEA } & P4 & СTCСTCCCACCCTCTCCCAggTTgTCCTCCCTTCACCTgT & 71.8 \\
\hline & P5 & gAgCAggAgCCTCTgCCAggggATgCACCATCTgTggggA & 73.7 \\
\hline & P6 & gCTATC TAgATCACAgCCCTgTCCTACCCAggAgACCCCAgCTCC & 71.1 \\
\hline & P7 & gCTATCTAgACAgCCCTgTCCTACCCAggAgACCCCAgCTCC & 71.0 \\
\hline & P1 & TACgTCTAgAAAAgTCgAggCggAggTACAAATTgACAggTTAATTACA & 65.7 \\
\hline & $\mathrm{P} 2$ & ggCAgACTTCAAAgCCTTCAAACCTATgTAACACAACAAC & 63.9 \\
\hline & P3 & TAATCAggATTAAATggCCTTggTATgTTggCTCggCT & 65.3 \\
\hline \multirow[t]{5}{*}{ SARS } & P4 & CATTgCTggACTAATTgCCATCgTCATggTTACAATCTI & 63.7 \\
\hline & P5 & gCTAAAgCTITTAAgTCATgCAACAAAgCAAgATTgTAACCATgACgA & 64.1 \\
\hline & P6 & TggCAATAgTCCAgCAATgAAgCCgAgCCAAACATACCA & 67.7 \\
\hline & P7 & AggCCATTAATCCTgATTAgTTgTgTgTTACATggTT & 61.7 \\
\hline & P8 & TgAAggCTITgAAgTCTgCCTgTAATTAACCTGgTCAATTा & 63.2 \\
\hline
\end{tabular}

days before inoculation with colon cancer CT26/CEA cells. As per the data shown in Figure 3, the tumor volume was significantly lower in the pCEA-SARS-CoV group $(p<0.05)$ as compared with the pCEA group, indicating that the SARS fragment provided sufficient protection against the development of tumors.

Regarding the therapeutic effects of the vaccines, mice were inoculated with $1 \times 10^{5} \mathrm{CT} 26 / \mathrm{CEA}$ and 4 days later orally immunized with $1 \times 10^{8} \mathrm{~S}$. typhimurium transformed with pCEA, or pCEA-SARS-CoV in $20 \mu \mathrm{l}$ PBS, while the negative control group was fed with $20 \mu \mathrm{l}$ PBS. While therapy with pCEA was not able to induce responses to reduce the growth of tumors in comparison with the control group, mice in the CEA-SARS-CoV group exhibited significantly less tumor growth, indicating that the SARS fragment provided adequate protection against CT26/CEA (Figure 4, $p<0.05$ in comparison with the control and the pCEA group).

\section{Discussion}

Our results demonstrated that fusion peptides composed of a highly-immunogenic epitope and a poorly-immunoreactive tumor-associated antigen can effectively activate host immunity, and furthermore, using the

Table 2 PCR primers used to synthesize CEA and SARS peptides.

\begin{tabular}{|c|c|c|c|}
\hline H2-Kd nonamer & Amino acid & Score & References \\
\hline CEA & SAPPHRWCI & 16 & NCBI accession No. Z21218 \\
\hline SARS & WYWLLGFIA & 21 & NCBI accession No. AB263618 \\
\hline Listeria LLO $_{91-99}$ & GYKDGNEYI & 24 & Nakamura et al., 2003 \\
\hline Listeria $\mathrm{p} 60_{217-225}$ & KYGVSVQDI & 27 & Bouwer et al., 1996 \\
\hline $\mathrm{EGFP}_{200-208}$ & HYLSTQSAL & 27 & Gambotto et al., 2001 \\
\hline
\end{tabular}

Predicted by the SYFPEITHI algorithm. prediction algorithm SYFPEITHI to enhance the affinity of antigen presentation, the resulting fusion peptides provoked even stronger immune responses. In addition, this study also provided new insight into tumor-specific

a.

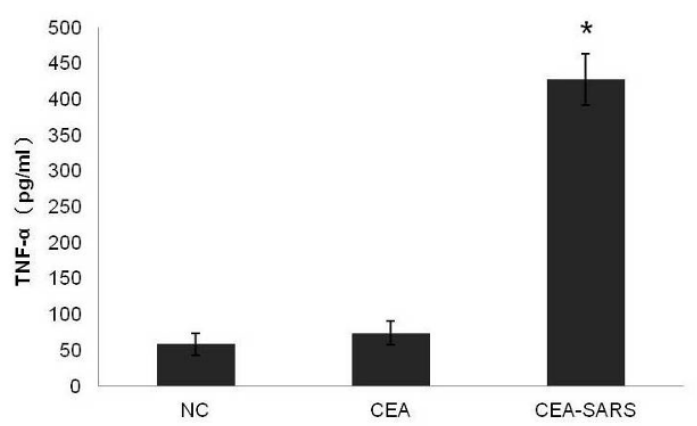

b.

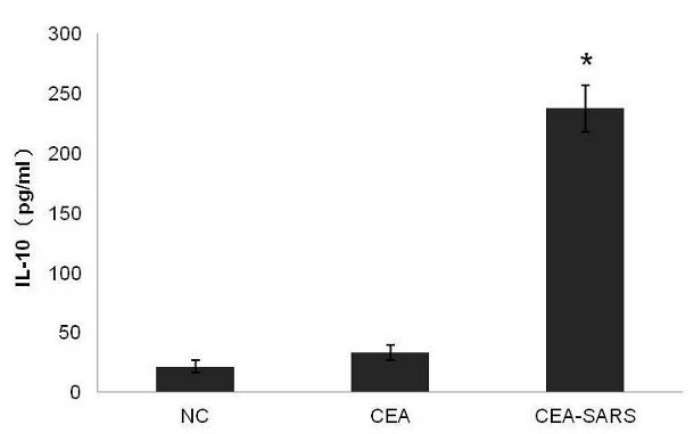

Figure 2 Serum cytokine profiles of BALB/C mice orallyvaccinated with CEA-SARS-derived epitopes. Sera samples were collected for in vivo cytokine detection, including (a) TNF- $\alpha$ and (b) IL-10. ${ }^{*}, p<0.05$ in comparison with the control and the PCEA group). 


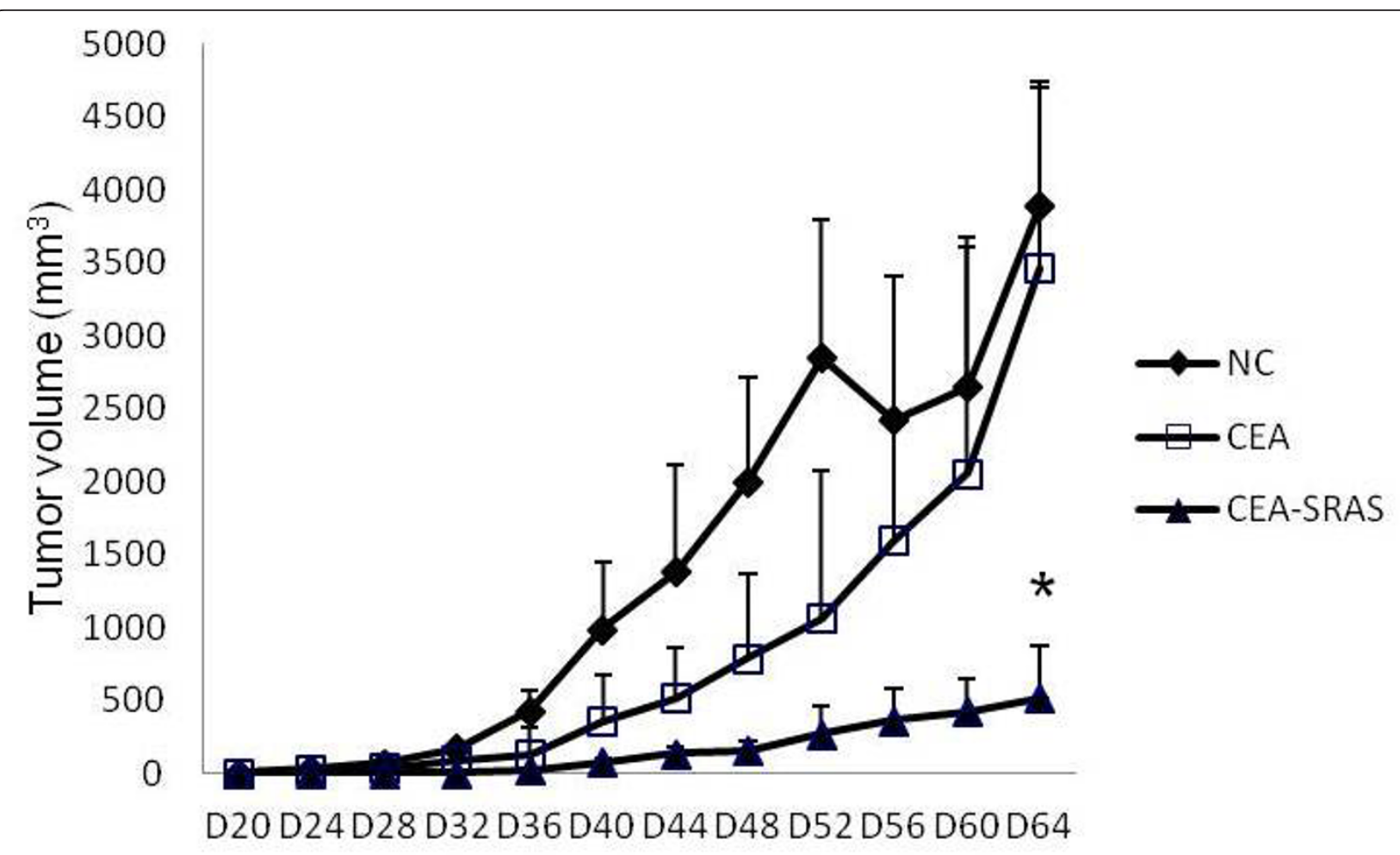

Figure 3 In vivo tumor development of BALB/c mice after oral vaccination. Five BALB/C mice in each treatment group were inoculated with CT26/CEA cells 21 days after vaccination with pCEA- and PCEA-SARS-transformed S. typhimurium and the tumor volume in the mice was observed. (*, $p<0.05$ in comparison with the control and the PCEA group).

immunity. By combining SARS-CoV epitope, a tumor unrelated antigenic fragment, with CEA, we finally demonstrated this could be a promising anti-cancer strategy through effectively increasing both Th1 and Th2 cytokines and decreasing tumor volume.

The DNA vaccine, though usually injected intramuscularly, can be administered orally in an animal model with bacteria, and it has been observed that some display preferential replication or preferential accumulation in the tumor microenvironment [8]. Among all bacteria, S. typhimurium is one of the most utilized to carry therapeutic transgenes. Attenuation of $S$. typhimurium with partial gene modifications does not affect its ability to achieve high tumor/normal tissue ratios in mouse models, and the bacteria maintain their capacity to inhibit the growth of both subcutaneous tumors and lung metastatic diseases [8]. Other modifications have also been reported; for example, the strain used in this study was attenuated aromatic acid-dependent (aro) S. typhimurium, which has been well-characterized as a carrier of various heterogeneous antigens $[9,10]$. These vaccine strains are capable of colonizing the gut-associated lymphoid tissues (Peyer's patches) and secondary lymphatic tissues including the spleen and liver following oral administration in mice, eliciting mucosal, humoral and cell-mediated immune responses [11]. Previous studies have proven the lipopolysaccharides on the Salmonella surface enable CD14+ monocytes to secrete TNF- $\alpha$, IL1 and IL-6 [12,13]. Also S. Typhimurium could deliver directly into the cytosol of macrophages and dendritic

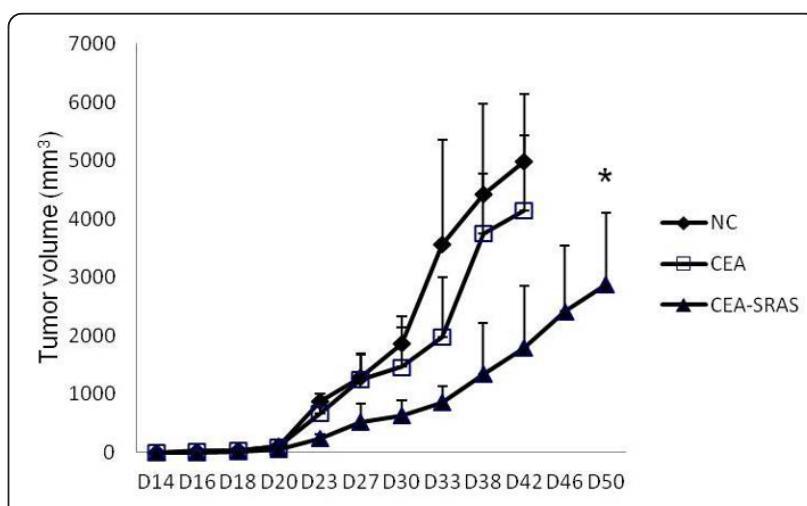

Figure 4 Therapeutic effect of CEA-SARS-derived epitopes on tumor establishment. BALB/C mice were inoculated with CT26/ CEA cells on day 0 . Four days later, the mice were fed with PCEAand PCEA-SARS-transformed S. typhimurium once per week (5 mice/ group), and the subsequent tumor volume in the mice was observed. $\left(^{*}, p<0.05\right.$ in comparison with the control and the pCEA group). 
cells resulting in vigorous antigen-specific $\mathrm{CD} 8+\mathrm{T}$ cell priming and the induction of protective immunity against viruses, bacteria and tumors $[14,15]$.

Variations in the cytokine profiles arose after vaccination with pCEA-SARS-CoV. As a highly-immunogenic peptide, treatment with SARS-containing epitopes has elicited a striking increase in TNF- $\alpha$ expression as compared with the negative control and the CEA group (Figure 2a). TNF$\alpha$ is a proinflammatory and Th1 cytokine, and as indicated by Austin and colleagues, TNF- $\alpha$, IFN- $\gamma$, and IL- 2 can be used to define cytotoxic T lymphocytes and Th1 effector populations [16]. Reducing local expression of TNF- $\alpha$ was found to be associated with B16-F10 melanoma outgrowth in mice while s.c. administrating TNF- $\alpha$ significantly suppressed primary tumor growth of melanoma [17]. IL-10, a typical Th2 cytokine, was enhanced in the pCEA-SARS$\mathrm{CoV}$ group as compared with the negative control and the CEA group (Figure $2 \mathrm{~b}$ ). Though most often known as a Th2 cytokine, IL-10 is actually a pleiotropic cytokine with anti-inflammatory, immunosuppressive, and immunostimulatory properties [18], and exerts immunostimulatory effects on B cells, cytotoxic T-cell development and thymocytes [19]. As indicated by Wogensen et al., expression of an IL-10 transgene by insulin-producing pancreatic cells led to the accelerated onset of diabetes in NOD mice $[20,21]$, with no inhibition of immune-mediated destruction of islets [22].

In the study of Moritani et al., NOD (Non-obese diabetic) mice expressing an IL-10 transgene in glucagonproducing pancreatic cells also developed accelerated diabetes [23]. Consequently, the expression of IL-10 in our model may exert not only a Th2 effect but also a Th1 effect. In general, the CEA antigen only induces a Th2 response, whereas the addition of a SARS fragment helps to induce and enhance both Th1 and Th2 responses. Th1 is more famous for its antitumor effects, although Th2 responses have been reported in autologous tumors, T-cells from patients with indolent nonHodgkin lymphomas frequently exhibiting an activated but apoptosis-prone phenotype [24]. It may seem that the Th2 response might downregulate the effect of a Th1 response or contribute less to the antitumor activity, but Th2-dominated antitumor immunity has also been observed in some models. As reported by Chu et al. [25], their DNA vaccine, which comprised a modified core peptide of mucin1 (PDTRP) and a GM-CSF coding sequence at the $\mathrm{C}$-terminus, induced better protection against tumor challenge, and that protection was correlated with type 2 immune responses manifested by an increased IgG1 to IgG2a antibody ratio and greater induction of GATA-3 and IL-4 mRNA than of T-bet and IFN- $\gamma$ mRNA in spleen cells of vaccinated mice.

The enhancement of Th1 and Th2 in the SARS-fragment-containing treatment groups indeed displayed better immunoactivity and protective effects in CT26/ pCEA-bearing mice. In comparison with the negative control group, no significant differences in tumor volume were observed in mice immunized with CEA alone, while the tumor volume was found to be smaller in the pCEA-SARS-CoV group in the protection and therapy assays (Figure $3 \& 4$ ). These results may indicate that enhancement of the affinity of antigen presentation by the prediction algorithm increased the immunogenicity of the CEA-SARS peptide.

\section{Conclusions}

This study has demonstrated a potential strategy to overcome traditional obstacles in TAA-specific tumor therapy, and has shown that it is possible for foreign SARS fragments to improve the anti-tumor efficacy of tumor vaccines against endogenous tumor antigens. Application of these kinds of highly-immunogenic peptides and the enhancement of peptide affinity through prediction software could serve as a platform to elicit powerful host immunity and eliminate tumor growth.

\section{Methods}

\section{Cell culture}

All cell lines were obtained from the American Type Culture Collection (VA, USA). CT26 (mouse colon cell, ATCC: CRL-2638), PT67 (mouse retrovirus-packaging cell line, ATCC: CRL-12284), and BALB/3T3 (mouse embryo fibroblast, ATCC: CCL-163.2) were cultured in RPMI media supplemented with $2 \mathrm{mM}$ L-glutamine, 50 $\mu \mathrm{g} / \mathrm{ml}$ of Penicillin/Streptomycin (P/S) and $10 \%$ heatinactivated fetal bovine serum (FBS) (Biological Industries, Haemek, Israel) at $37^{\circ} \mathrm{C}$ under $5 \% \mathrm{CO} 2 . \mathrm{CT} 26 /$ CEA cells were generated by transfecting retroviral vectors (pMSCV neo) carrying CEA DNA fragments (NCBI accession No. Z21818, nucleotide (nt.) CEA 10804 10938, $132 \mathrm{bp}$ ). CT-26 transfectant cells (CT26/CEA) were obtained by G418 (Protech, Taiwan) selection and maintained in the RPMI growth medium described above.

\section{Prediction of H2-Kd-immunogenic amino-acid peptides}

A fragment derived from the SARS coronavirus (SARSCoV) (NCBI accession No. AB263618) that has proved to be effective in eliciting IFN- $\gamma$ secretion in humans [6] was selected for use in this study and fused with CEA, a low-immunogenic peptide for peptide-binding prediction. The SYFPEITHI scoring system [26] was then utilized to predict the affinity of CEA and CEA-SARS-CoV peptides towards binding to $\mathrm{BALB} / \mathrm{c} \mathrm{H} 2-\mathrm{Kd}$ molecules.

\section{Construction of vectors for CEA and CEA-SARS-CoV gene expression}

The eukaryotic expression plasmid pAAV-MCS was chosen for gene insertion and expression under the 
control of a CMV promoter. Gene fragments containing CEA and SARS-CoV were both artificially synthesized by polymerase chain reaction (PCR) using a series of sense and antisense primers designed precisely according to the above two gene sequences (Table 1), which provided suitable restriction enzyme sites. Because the antisense sequences overlapped with the sense sequences for $20 \mathrm{bps}$ in the designed primers, they will base-pair with each other. The CEA fragment with the restriction enzyme sites EcoRI 5' and XbaI 3' and pAAV-MCS were both digested with EcoRI and XbaI and then ligated to form PCEA. The SARS fragment with XbaI '5' and HindIII 3' and pCEA were both digested with $\mathrm{XbaI}$ and HindIII and then ligated to form pCEA-SARS-CoV.

\section{Oral infection of BALB/c mice with Salmonella}

Plasmids were electroporated into the Salmonella vaccine vector (S. typhimurium strain SL3261) at $2.5 \mathrm{mF}, 2.5 \mathrm{kV}$, and $200 \Omega$ for $4 \sim 5 \mathrm{msec}$, and quantification of the bacteria was performed by plating serial dilutions in $L B$ plates. Six-eight-week-old female BALB/c mice were purchased from the National Laboratory Center (Taipei, Taiwan) and housed in a temperature- and light-controlled (12L:12D) environment at the Animal Maintenance Facility of National Chiao Tung University (NCTU) (HsinChu, Taiwan). The institutional animal care and use committee of NCTU approved this study before its start. Mice in the treatment groups were immunized with $1 \times$ $10^{8}$ attenuated S. typhimurium (in $20 \mu \mathrm{l}$ PBS) transformed with the different vectors (pCEA and pCEASARS-CoV) three times in two weeks, while the negative control group was fed with $20 \mu \mathrm{l}$ PBS alone.

\section{ELISA}

To evaluate the cytokine profiles of the mice after immunization with pCEA- and pCEA-SARS-CoV-transformed $S$. typhimurium, sera samples were collected at day 14 and assayed for TNF- $\alpha$ and IL-10 expression by standard ELISA ( $\&$ \& , MN, USA) in compliance with the manufacturer's instructions.

\section{Vaccine protection assay}

For the vaccine protection assay, 6-8-week-old BALB/c mice were immunized using the protocol described in the previous section. One week after the last booster injection, mice were inoculated with $5 \times 10^{5}$ CT26/CEA cells and the tumor growth pattern was measured every 4 days. Tumor volume was calculated using the formula volume $=$ length $\times$ width $\times$ height.

\section{Vaccine therapeutic assay}

To evaluate the tumor therapy, BALB/c mice were inoculated with $1 \times 10^{5}$ CT26/CEA cells (in $200 \mu \mathrm{l} \mathrm{PBS/}$ mouse) and orally immunized with $1 \times 10^{8}$ S. typhimurium (in $20 \mu \mathrm{l} \mathrm{PBS} /$ mouse) 4 days later, followed by reimmunization once a week after the first injection.

\section{Statistical analysis}

Results are expressed as mean \pm SE. Student's $t$ test was applied to compare the effects of treatment in the different groups, and the Mantel-Cox significance test was employed to analyze the survival rate. A value of $p<$ 0.05 was considered significant.

\section{Author details}

${ }^{1}$ School of Veterinary Medicine, National Taiwan University, Taipei, Taiwan. 2Department of Biological Science and Technology, National Chiao-Tung University, Hsin-Chu, Taiwan. ${ }^{3}$ Department of Food Science, National Taiwan Ocean University, Keelung, Taiwan. ${ }^{4}$ Department of Nursing, Tzu Chi University, Hualien, Taiwan.

\section{Authors' contributions}

KWL and CSL conceived the study aims and design, contributed to the systematic review and data extraction, performed the analysis and interpreted the results. SHK drafted the manuscript and performed the experiments. YCC, CHL, YTH, and SCY assisted in doing experiments. CJW and RPL contributed to the revision of the manuscript. All authors have read and approved the final version of this paper.

\section{Competing interests}

We (authors in this manuscript) declares that we have no significant competing financial, professional or personal interests that might have influenced the performance or presentation of the work described in this manuscript.

Received: 14 November 2011 Accepted: 3 February 2012

Published: 3 February 2012

\section{References}

1. Reuschenbach M, von Knebel Doeberitz M, Wentzensen N: A systematic review of humoral immune responses against tumor antigens. Cancer Immunol Immunother 2009, 58:1535-1544.

2. Hodge JW, Greiner JW, Tsang KY, Sabzevari H, Kudo-Saito C, Grosenbach DW, Gulley JL, Arlen PM, Marshall JL, Panicali D, Schlom J: Costimulatory molecules as adjuvants for immunotherapy. Front Biosci 2006, 11:788-803.

3. Hallermalm K, Johansson S, Brave A, Ek M, Engstrom G, Boberg A, Gudmundsdotter L, Blomberg P, Mellstedt H, Stout R, et al: Pre-clinical evaluation of a CEA DNA prime/protein boost vaccination strategy against colorectal cancer. Scand J Immunol 2007, 66:43-51.

4. Dai S, Wei D, Wu Z, Zhou X, Wei X, Huang H, Li G: Phase I clinical trial of autologous ascites-derived exosomes combined with GM-CSF for colorectal cancer. Mol Ther 2008, 16:782-790.

5. Ojima T, Iwahashi M, Nakamura M, Matsuda K, Nakamori M, Ueda K, Naka T, Ishida K, Primus FJ, Yamaue H: Successful cancer vaccine therapy for carcinoembryonic antigen (CEA)-expressing colon cancer using genetically modified dendritic cells that express CEA and T helper-type 1 cytokines in CEA transgenic mice. Int J Cancer 2007, 120:585-593.

6. Wang $Y D$, Sin $W Y, X u G B$, Yang $H H$, Wong TY, Pang XW, He XY, Zhang HG, $\mathrm{Ng} \mathrm{JN}$, Cheng CS, et al: T-cell epitopes in severe acute respiratory syndrome (SARS) coronavirus spike protein elicit a specific T-cell immune response in patients who recover from SARS. J Virol 2004, 78:5612-5618.

7. Darji A, Guzman CA, Gerstel B, Wachholz P, Timmis KN, Wehland J, Chakraborty T, Weiss S: Oral somatic transgene vaccination using attenuated S. typhimurium. Cell 1997, 91:765-775.

8. Sznol M, Lin SL, Bermudes D, Zheng LM, King I: Use of preferentially replicating bacteria for the treatment of cancer. J Clin Invest 2000, 105:1027-1030. 
9. Dougan G, Hormaeche CE, Maskell DJ: Live oral Salmonella vaccines: potential use of attenuated strains as carriers of heterologous antigens to the immune system. Parasite Immunol 1987, 9:151-160.

10. Fagan PK, Djordjevic SP, Chin J, Eamens GJ, Walker MJ: Oral immunization of mice with attenuated Salmonella typhimurium aroA expressing a recombinant Mycoplasma hyopneumoniae antigen (NrdF). Infect Immun 1997, 65:2502-2507.

11. Molecular and Clinical Aspects of Bacterial Vaccine Development. Edited by: Hormaeche DAAAACE 1995

12. Proskuryakov SY, Gabai VL: Mechanisms of tumor cell necrosis. Curr Pharm Des 2010, 16:56-68.

13. Wright SD, Ramos RA, Patel M, Miller DS: Septin: a factor in plasma that opsonizes lipopolysaccharide-bearing particles for recognition by CD14 on phagocytes. J Exp Med 1992, 176:719-727.

14. Russmann H, Shams H, Poblete F, Fu Y, Galan JE, Donis RO: Delivery of epitopes by the Salmonella type III secretion system for vaccine development. Science 1998, 281:565-568.

15. Walker $\mathrm{EB}$, Disis ML: Monitoring immune responses in cancer patients receiving tumor vaccines. Int Rev Immunol 2003, 22:283-319.

16. Austin LM, Ozawa M, Kikuchi T, Walters IB, Krueger JG: The majority of epidermal T cells in Psoriasis vulgaris lesions can produce type 1 cytokines, interferon-gamma, interleukin-2, and tumor necrosis factoralpha, defining TC1 (cytotoxic T lymphocyte) and TH1 effector populations: a type 1 differentiation bias is also measured in circulating blood T cells in psoriatic patients. J Invest Dermatol 1999, 113:752-759.

17. Nakasone YFM, Matsushita T, Hamaguchi Y, Huu DL, Yanaba M, Sato S, Takehara K, Hasegawa M: Host-Derived MCP-1 and MIP-1a Regulate Protective Anti-Tumor Immunity to Localized and Metastatic B16 Melanoma. The American journal of pathology 2011.

18. Moore KW, de Waal Malefyt R, Coffman RL, O'Garra A: Interleukin-10 and the interleukin-10 receptor. Annu Rev Immunol 2001, 19:683-765.

19. Conti P, Kempuraj D, Kandere K, Di Gioacchino M, Barbacane RC, Castellani ML, Felaco M, Boucher W, Letourneau R, Theoharides TC: IL-10, an inflammatory/inhibitory cytokine, but not always. Immunol Lett 2003, 86:123-129.

20. Wogensen L, Huang $X$, Sarvetnick N: Leukocyte extravasation into the pancreatic tissue in transgenic mice expressing interleukin 10 in the islets of Langerhans. J Exp Med 1993, 178:175-185.

21. Wogensen L, Lee MS, Sarvetnick N: Production of interleukin 10 by islet cells accelerates immune-mediated destruction of beta cells in nonobese diabetic mice. J Exp Med 1994, 179:1379-1384.

22. Lee MS, Wogensen L, Shizuru J, Oldstone MB, Sarvetnick N: Pancreatic islet production of murine interleukin-10 does not inhibit immune-mediated tissue destruction. J Clin Invest 1994, 93:1332-1338.

23. Moritani M, Yoshimoto K, Tashiro F, Hashimoto C, Miyazaki J, li S, Kudo E, Iwahana H, Hayashi Y, Sano T, et al: Transgenic expression of IL-10 in pancreatic islet A cells accelerates autoimmune insulitis and diabetes in non-obese diabetic mice. Int Immunol 1994, 6:1927-1936.

24. Anichini A, Mortarini R, Romagnoli L, Baldassari P, Cabras A, Carlo-Stella C, Gianni AM, Di Nicola M: Skewed T-cell differentiation in patients with indolent non-Hodgkin lymphoma reversed by ex vivo T-cell culture with gammac cytokines. Blood 2006, 107:602-609.

25. Chu Y, Xia M, Lin Y, Li A, Wang Y, Liu R, Xiong S: Th2-dominated antitumor immunity induced by DNA immunization with the genes coding for a basal core peptide PDTRP and GM-CSF. Cancer Gene Ther 2006, 13:510-519.

26. Rammensee $H G$, Friede T, Stevanoviic S: MHC ligands and peptide motifs: first listing. Immunogenetics 1995, 41:178-228.

doi:10.1186/1480-9222-14-2

Cite this article as: Lin et al:: Enhancement of anti-murine colon cancer immunity by fusion of a SARS fragment to a low-immunogenic carcinoembryonic antigen. Biological Procedures Online 2012 14:2.

\section{Submit your next manuscript to BioMed Central and take full advantage of:}

- Convenient online submission

- Thorough peer review

- No space constraints or color figure charges

- Immediate publication on acceptance

- Inclusion in PubMed, CAS, Scopus and Google Scholar

- Research which is freely available for redistribution

Submit your manuscript at www.biomedcentral.com/submit
Biomed Central 